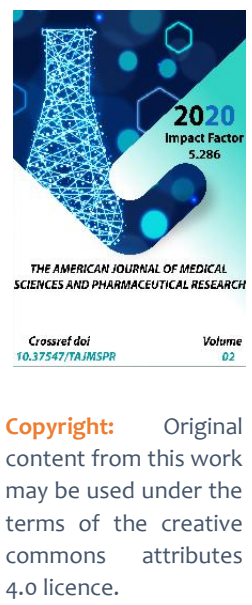

\title{
Morphological Features Of Microvascular Tissue Of The Brain At Hemorrhagic Stroke
}

\author{
Kamalova Malika Ilkhomovna \\ Assistant To The Department Of Human Anatomy And Surgical Topography Of The Samrkand \\ State Medical Institute, Uzbekistan \\ Islamov Shavkat Eriyigitovich \\ Doctor Of Medical Sciences, Department Of Forensic Examination And Pathological Anatomy, \\ Samarkand Medical Institute, Uzbekistan \\ Khaidarov Nodir Kadyrovich \\ Doctor Of Medical Sciences, Department Of Nervous Diseases And Physiotherapy Of Tashkent \\ State Dental Institute, Uzbekistan
}

\section{ABSTRACT}

Hemorrhagic stroke is less common than ischemic stroke and is heavier and in most cases has an adverse outcome in the form of death of a patient or permanent disability. Recently, there has been a tendency to increase the frequency of $\mathrm{Gl}$, which is increasingly found at a younger age. $\mathrm{Gl}$ is based on a rupture of blood vessels, the development of hemorrhage and as a consequence - the formation of cerebral edema, ischemia of brain tissue, violation of the integrity of GBS, imbalance of the neuroimmune system, as well as violation of the function of other systems and organs.

\section{KEYWORDS}

Brain tissue microvessels, hemorrhagic stroke, hemorrhagic zones, arterioles and precapillaries, microhematome

\section{INTRODUCTION}

Currently, the high frequency of strokes can be caused by the lack of an effective primary and secondary prevention system. Clarification of this issue is associated with a detailed study of risk factors, the course and prevention of stroke in our country. Comparatively little study remains on the implementation of secondary prevention of stroke, especially with its different subtypes, which makes it relevant to further research in this direction. Lethal 
outcome of stroke in different countries is 1.31.8 per 1000 people per year, which is 1.5 times older with repeated stroke. In recent decades, the idea of pathogenetic subtypes of strokes and the criteria for their diagnosis has been formed, and certain difficulties arise in tanatological practice. Therefore, the identification of characteristic morphological changes in brain stroke, as well as the creation of a classification of strokes on the basis of this representation are extremely important tools for the choice of tactics for their treatment and secondary prevention, the definition of shortand long-term prognosis, standardization in clinical and epidemiological studies.

\section{RESEARCH OBJECTIVE}

To study pathomorphological changes of arteries, arteriol of the microcirculatory channel of the brain during hemorrhagic stroke.

\section{RESEARCH MATERIALS AND METHODS}

As a material, a retrospective analysis of 50 protocols of pathological and anatomical research conducted at the Tanatological Department of the Republican Pathological Anatomical Center in the period 2018-2020 was carried out. Macro- and microscopic examination of the brain of intracerebral arteries and microcirculation channel vessels (MCR) was carried out. The study of the brain determined the size and localization of intracerebral hematomas, the presence of a blood rupture into the ventricular system, the severity of cerebral edema, dislocation and compression of its trunk. Small hemorrhages, foci of perivascular edema, spongiform condition of white matter were considered. Microscopic examination of the brain was carried out in histological preparations contained in paraffin. Which were stained with hematoxylin and eosin, by the methods of van
- Gizon (determination of collagen fibers and myocytes in vessels), Weigert (determination of elastic fibers in vessels). Particular attention was paid to the microcirculatory vessels within the hematoma, in the perifocal zone, as well as at a distance from the hematoma.

\section{RESEARCH RESULTS AND THEIR DISCUSSION}

All diagnosed acute cerebral circulation disorder hemorrhagic type. Among them 38 men (75.0\%) aged 20 to 72 years, 12 women (25.0\%) aged 33 to 65 years.

In case of cerebral blood circulation disturbance in the form of hemorrhagic stroke, dystrophic, dysregenerative and inflammatory processes develop in the vascular system, characterized by endothelium, basal and elastic membrane, muscular layer and adventilation with subsequent violation of permeability of the wall, swelling of the vascular wall and surrounding tissue, hemorrhage. In this case, pathomorphologically there is swelling, rejection of the endelium, denudation, disorganization, mucoid and fibrinoid swelling and fibrinoid necrosis of the basal membrane. Disorganization, swelling, delamination and lysis of fibrous structures are observed on the side of the elastic membrane. The muscle layer of the vascular wall reacts by proliferation and activation of smooth muscle cells and their germination into other layers of the vascular wall. Around the brain arteries, the adventiation is gentle and represented by single connective tissue cells and loose fibrous structures. Therefore, in a hemorrhagic stroke, adventilation of brain vessels is subject to edema, mucoid and fibrinoid swelling with the development of an inflammatory reaction involving connective tissue cells and brain glia. Microscopic examination of brain tissue during hemorrhagic stroke showed that the main pathomorphological changes are observed in 
the wall of all vascular components of the ICR. In brain tissue arteries and arterioles are found on the surface cortical layers and under the meningeal membranes. In case of hemorrhagic stroke it is noted that in the artery wall flattening, desquamation of endothelial cells and the formation of surface defects in the form of absence of basal membrane covering cells. The elastic membrane is thickened due to loosening and corrugation of individual fibrous bundles (Fig. 1). Such loosening of the elastic fibers leads to separation of the muscle layer of the vessel wall. Smooth-muscular layer of artery wall has uneven thickness due to hypertrophy of some areas and atrophy of other areas. In the circumference of the artery in the cerebral tissue is formed an oedema space with decay and destruction of brain matter.

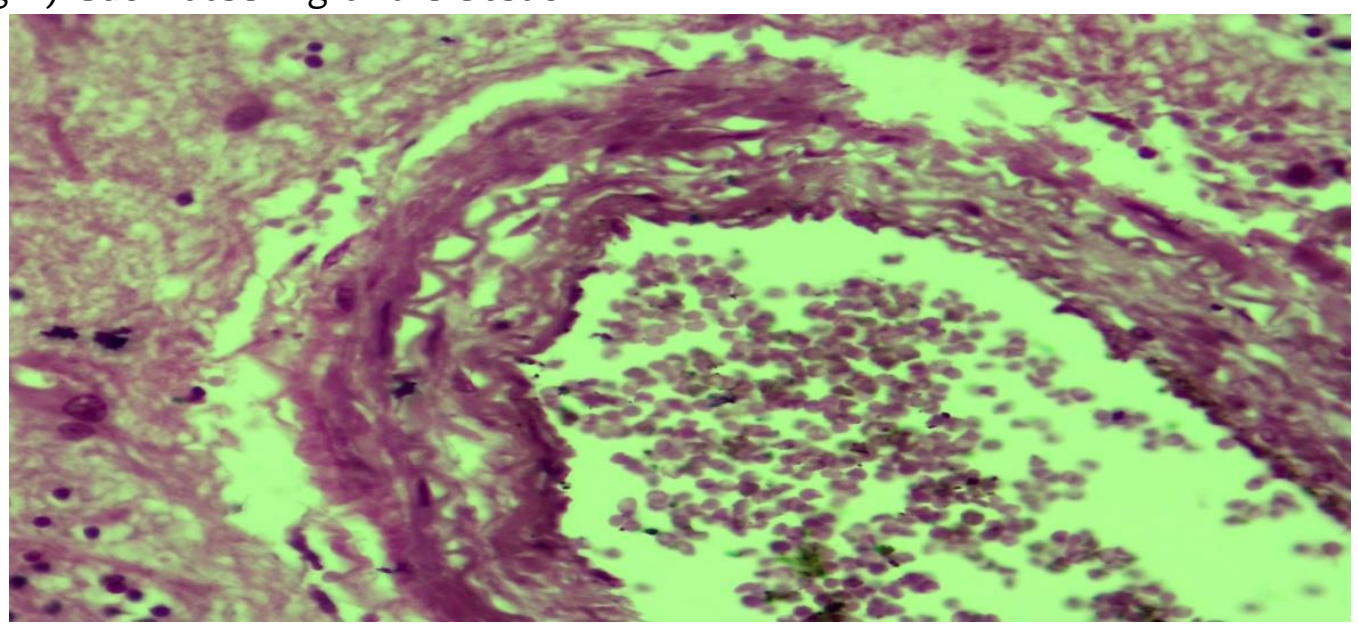

Fig.1. Loosening of artery wall layers in case of hemorrhagic stroke. Coloring: hematoxylineosin. Enlarged: $10 \times 40$.

Study of the artery of the hemorrhage zone noted that the artery is surrounded by a focus of hemorrhage in the form of infiltration of brain matter by red blood cells and single leukocytes, due to which the brain matter in a state of edema, decay and destruction. Cellular elements of brain tissue, in particular clay in a state of hypertrophy and hyperchromasia, some of them have been transformed into large macrophage cells. The artery wall is unevenly thinned, with endothelial cells flattened, an elastic membrane in a state of fibrinoid swelling, single muscle cells and rejection of elastic membranes (Fig. 2). 


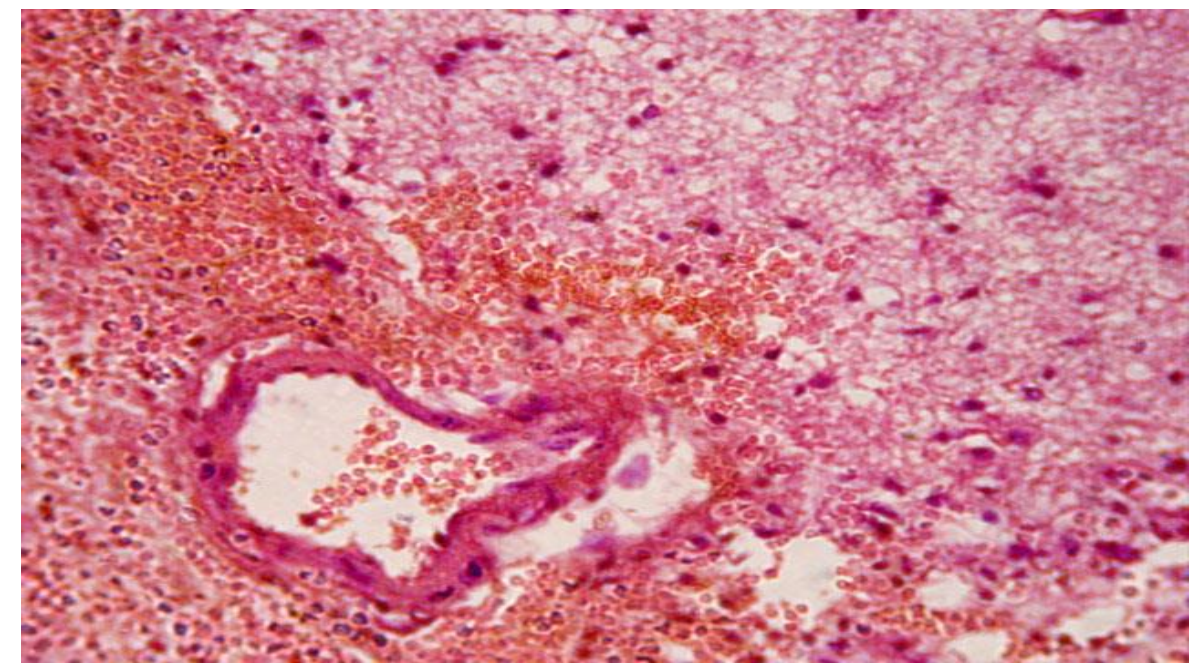

Fig.2. Hemorrhagic stroke hemorrhage in artery circumference in brain tissue.Coloring:

hematoxylin- eosin. Enlarged: $10 \times 40$.

In some arteries there is a rupture of the wall and hemorrhage in the perivascular space with the formation of microhematom (Fig. 3). In this case, the wall of the artery determines the complete disorganization and destruction of fibrous structures in the form of fibrinoidnecrosis, rupture. Due to this there was a perivascular hemorrhage, restriction of the blood mass by the adventitial tissue and the formation of microhematoma. Proliferations of adventicial cells and clay with the formation of proliferative inflammatory shaft were observed around the hematoma from the brain tissue.

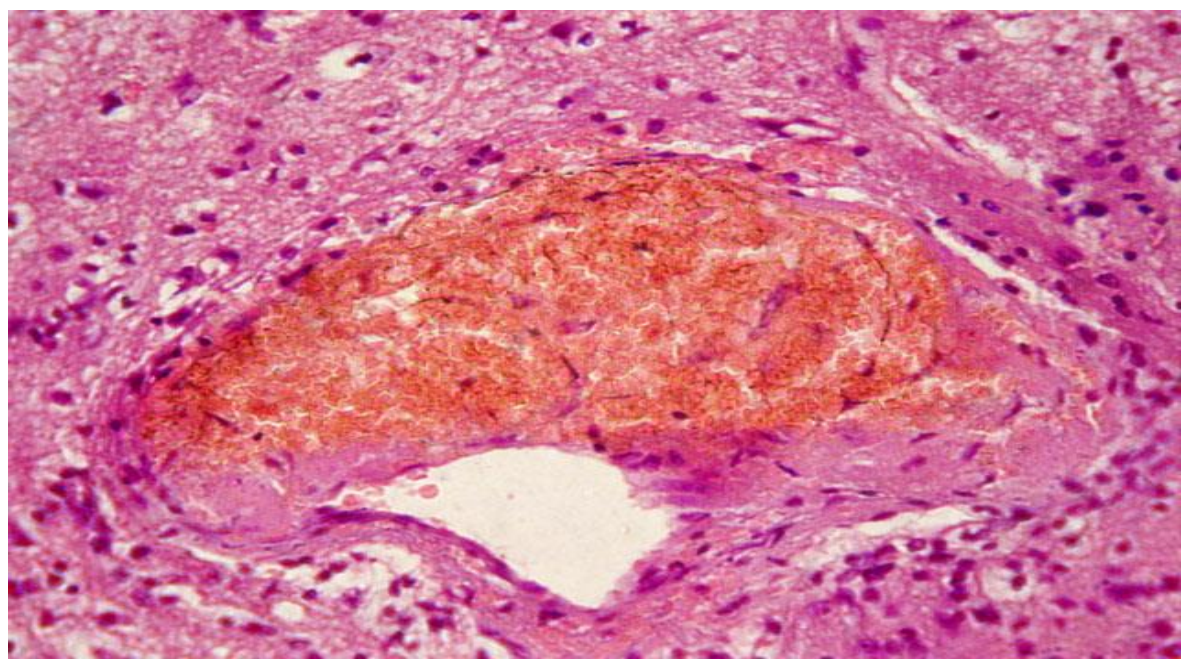

Fig. 3.Periarterial hemorrhage with the formation of microhematoma. Coloring: hematoxylin- eosin. Enlarged: $10 \times 40$.

Arterioles are defined in deeper parts of brain tissue with a diameter of 20 to 35 microns and they are localized directly in the thickness of brain tissue and there is almost no adventitial 
envelope around them. In hemorrhagic stroke, most arterioles are dilated in round or oval shape. The wall consists of an endothelium, a thin basal and elastic membrane, and one or two layers of smooth-muscular cells are defined at the periphery. Endothelial lining is thin, it is difficult to determine the basal and elastic membrane in the state of edema, myxomatosis, individual foci are subjected to fibrinoid swelling. Due to the violation of the integrity of the arteriol wall, there is a perivascular hemorrhage in the form of bruising in the thickness of brain tissue (Fig. 4).

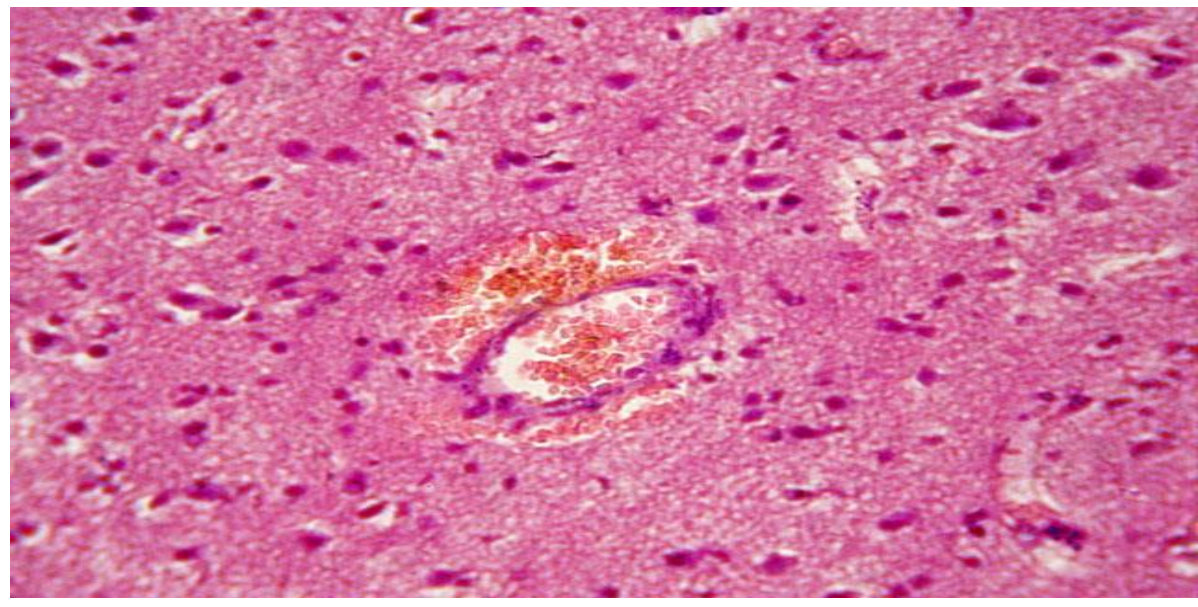

Fig.4.Arteriol with damage to the wall and bleeding into the surrounding tissue.Coloring:

hematoxylin- eosin. Enlarged: $10 \times 40$.

Arterioles and precapillaries are more severely damaged during hemorrhagic stroke than all intracerebral vessels. At the same time, the development of various pathological processes in the wall of these arteries is noted. In some arteries there is a pronounced damage of the inner layers of the wall in the form of endothelium desquamation, destruction of the basal and elastic membrane with hemorrhage in the vessel wall and around. Other arterioles show dilatation of the vessel, infiltration of the wall by inflammatory and glial cells (Fig. 5). 


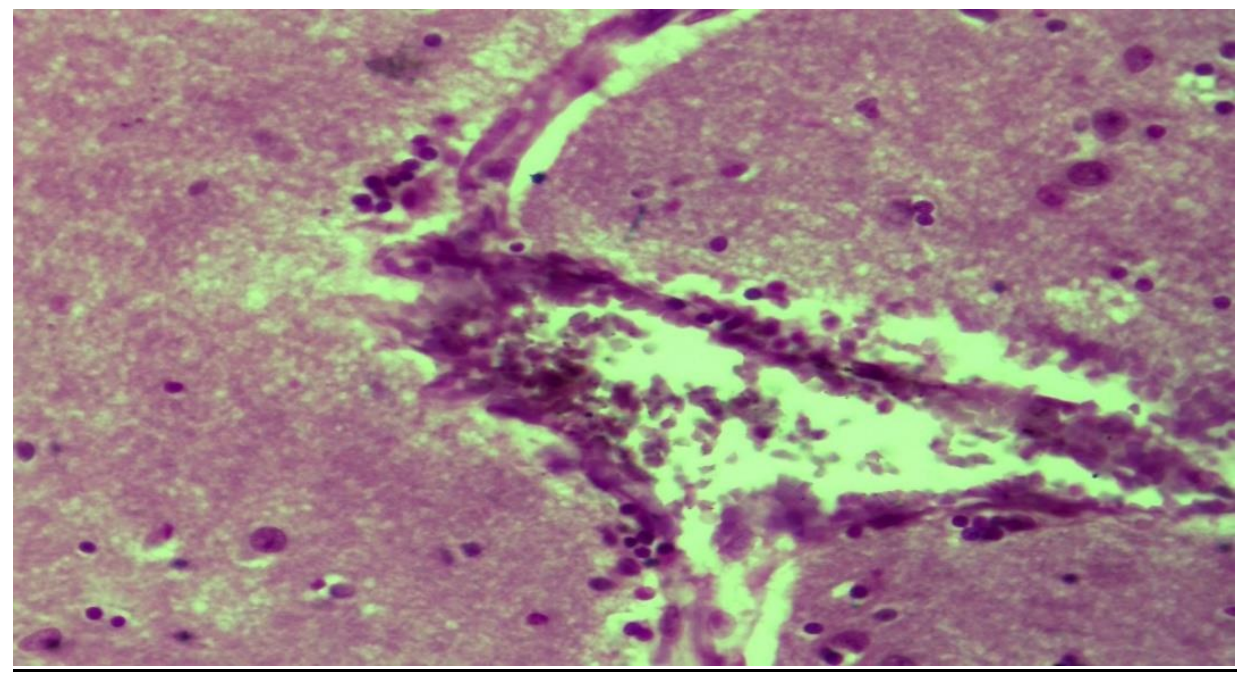

Fig. 5.Precapillaryarteriol in the state of dilatation and infiltration of the wall by inflammatory cells.Coloring: hematoxylin- eosin. Enlarged: $10 \times 40$.

Inflammatory cells tightly infiltrates all layers of the vessel wall, in some places leads to rupture and loosening and hemorrhage. Around these precapillaries marked the development of marked perivascular edema and loosening of brain tissue. At the mouth of the departing capillaries there is a focal inflammatory infiltration. In the adjacent areas of hemorrhage in the brain tissue is marked by marked edema and loosening of brain matter with the breakdown and homogenization of nerve fibers. Precapillaries of these zones are relatively narrowed, their wall is deformed, thickened and hyperchromic, in a circle there is perivascular hemorrhage with the breakdown of red blood cells (Fig. 6). Glial cells are activated as hypertrophy and hyperchromasia of nuclear structures.

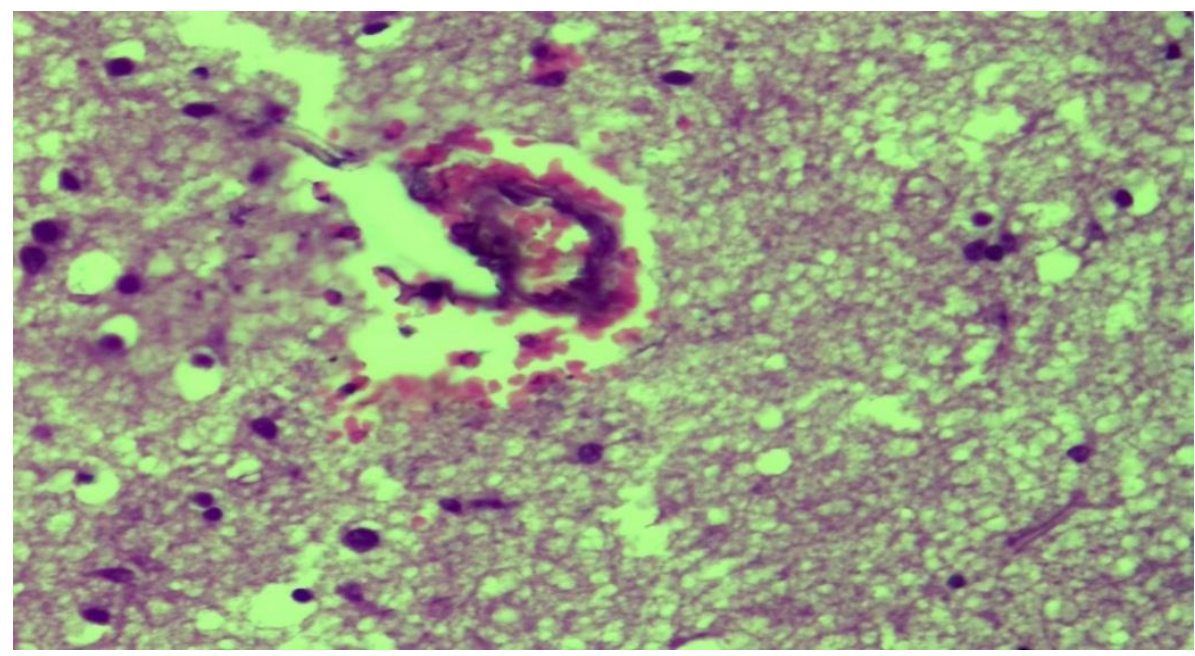

Fig. 6. Expressed edema and loosening of brain matter, perivascular hemorrhage.Coloring: hematoxylin- eosin. Enlarged: 10x40. 


\section{CONCLUSIONS}

Thus, the cerebral tissue is supplied with blood through the blood vessels departing from the arterial network located in the soft cerebral envelope. Since the arteries that supply the cortex and white matter are as if they are radiating from the brain surface to the ventricles, they can be called radial for the sake of brevity, histological examination is necessary to clarify the stage of brain stroke. In this case, the change in the nature of hemorrhagic component with the appearance of small or focal fresh hemorrhages in the focus of stroke should be taken into account when interpreting the results of the determination of the age of stroke. Pathomorphological changes cover all structural and functional levels of the arterial system of the brain, the most important of which are the vessels of the microcirculatory channel.

\section{REFERENCES}

1. Anufriev P.L., Gulevskaya T.S. Early histological signs of brain death syndrome in stroke (in Russian) // Vestnik of Russian Military Medical Academy. - - 2019. Tom67, No. 3. - C.19-21.

2. Suslina, Z.A. Brain circulation disorders: diagnostics, treatment, prevention / Z.A. Suslina, T.S. Gulevskaya, M.Yu. - Moscow: MEDpress-Inform, 2016. - 536.

3. Colonna M., Butovsky O. Microglia function in the central nervous system during health and neurodegeneration. // Annu. Rev. Immunol. - 2017. - №35 - P. 441468.

4. Cooke R.A.,Stewart B. Colour Atlas of Anatomical Pathology. -Churchill Livingstone. - 2004. - 300 p.

5. Donnan G.A., Fisher M., Macleod M., Davis S.M.Stroke(англ.)//The Lancet.-Elsevier,
2008. - May (vol. 371, no. 9624).- P. 1612-1623.

6. European Stroke Initiative recommendations for stroke management: update 2003 // Cerebrovasc. Dis. - 2003. - Vol. 16, - P.311-337.

7. Faustino J., Wang X., Johnson C.E., Klibanov A., Derugin N., Wendland M.F., et al. Microglial cells contribute to endogenous brain defenses after acute neonatal focal stroke. //J. Neurosci.- 2011. №31.- P.12992-13001.

8. Fumagalli S., Perego C., Ortolano F., De Simoni M.G. CX3 3 R1 deficiency induces an early protective inflammatory environment in ischemic mice. // Glia. 2013. - №61.P. 827-842. 